\title{
Diagnostic Value of Ultrasound-Guided Percutaneous Biopsy in the Evaluation of Cervical Lymph Node Lesions
}

\author{
Homagni Sikha Roy*, Qing Zhu, Chunxia Cheng, Yan Li, Xinchao Luo, Yi Mao and Yue Luo \\ Department of Ultrasound, Affiliated Traditional Chinese Medicine Hospital, Southwest Medical University, China
}

*Corresponding author: Homagni Sikha Roy, Department of Ultrasound, Affiliated Traditional Chinese Medicine Hospital

of Southwest Medical University, 182 Xianglin road, Longmatan district, Luzhou,Sichuan646000, China

\begin{tabular}{|c|}
\hline ARTICLE INFO \\
\hline Received: 蔧 July 09, 2020 \\
\hline Published: 幽 September 10, 2020 \\
\hline
\end{tabular}

Citation: Homagni Sikha Roy, Qing Zhu, Chunxia Cheng, Yan Li, Xinchao Luo, Yi Mao, Yue Luo. Diagnostic Value of UltrasoundGuided Percutaneous Biopsy in the Evaluation of Cervical Lymph Node Lesions. Biomed J Sci \& Tech Res 30(2)-2020. BJSTR. MS.ID.004924.

Keywords: Ultrasound guidance; Percutaneous biopsy; Cervical lymph node lesion

\begin{abstract}
Objective: To investigate the diagnostic value of ultrasound-guided percutaneous biopsy in the diagnosis of cervical lymph node lesions.

Methods: From January 2017 to August 2019, ultrasound-guided percutaneous biopsy, using clinical 16G and 18G biopsy needles, were used in 190 patients with cervical lymph node lesions. After taking out the target tissue and having a pathologically confirmed diagnosis, it was possible to determine whether the material was satisfactory or not. All patients underwent ultrasound-guided biopsy for pathological diagnosis and postoperative pathology confirmed the final diagnosis.
\end{abstract}

Results: The study found that 185 patients had satisfactory materials, reaching $97.37 \%$ of the satisfaction rate. For cervical lymph node lesions, Ultrasound-guided $16 \mathrm{G}$ needle biopsy achieved 100\% sensitivity, 96\% specificity, and 98\% accuracy, whereas Ultrasound-guided 18G needle biopsy achieved 100\% sensitivity, 94\% specificity, and $97 \%$ accuracy respectively. There was no significant difference noted between the two needle biopsies in the diagnosis of benign and malignant lesions $(\mathrm{P}>0.05)$; with $84 \%$ sensitivity, 93\% specificity, and $87 \%$ accuracy being significantly different $(\mathrm{P}<0.05)$.

Conclusion: Ultrasound-guided percutaneous biopsy is able to ensure a high accuracy of diagnosis and thereby reduce the occurrence of complications in patients with cervical lymph node lesions.

\section{Introduction}

In recent clinical practice, patients presenting with chief complaint of cervical lymphadenopathy[1] has been progressively increasing year after year and as a result, the main causes have been becoming more complicated. Therefore, clinical diagnosis of the benign and malignant lymph node lesions needs to be precise, as the development of clinical treatment programs are crucial[2]. Although clinically diagnosed cervical lymph node lesions, based on pathological diagnosis of excision biopsy, can achieve high diagnostic accuracy, yet traumatic, sampling difficulties and other issues are encountered. This article explores the diagnostic value of clinical ultrasound guided percutaneous biopsy for cervical lymph node lesions [3].Cervical lymphadenopathy simply refers to the disease or enlargement of the lymph nodes of the neck, and it's considered more of a physical sign or symptom rather than a diagnosis. There can be various reasons including degenerative, inflammatory or neoplastic. However healthy lymph nodes can be palpable in the axilla, neck and groin region. Numerous Infectious causes of cervical lymphadenopathy including most importantly HIV, Tuberculosis and Chicken pox exist. For malignant diseases, this lymphadenopathy can be either reactive or metastatic[4].

In case of malignancy being suspected, routine throat examination including mirror and endoscopy is performed. 
B-mode Ultrasound imaging of the cervical lymph node would depict morphology while the power Doppler would assess the vasculature[2].Cervical lymphadenopathy in suspected case of malignancies is diagnosed mostly using Fine needle aspiration cytology under ultrasound guidance[5]. It is quite effective procedure as while performing the percutaneous needle biopsy the doctor is not blind folded and because of the real time ultrasound imaging there is less chance of damaging any structures while performing the biopsy.

Lymphadenopathy when first suspected has to undergo tests for a definitive diagnosis. Ultrasound imaging of the lymph node would tell us whether there is enlargement or any other dimensional changes. But to deduce whether the enlargement or mass is benign or malignant, we need to perform a biopsy and test histologically and pathologically[6]. So, for that, if we can decide on the best diagnostic test to be the ultrasound guided percutaneous biopsy, then it would be advantageous[7].

\section{Materials and Methods}

\section{Study population}

From January 2017 to August 2019, clinical 16 G and 18 G biopsy needles were used in 190 patients with cervical lymph node lesions under ultrasound guided percutaneous biopsy. Of the 190 patients, 130 were male and 60 were females. Their age ranged from 5 to 75 years old (mean age $23.7 \pm 16.4$ years) and included 55 cases of pulmonary tuberculosis, 25 cases of combined pleurisy and 8 cases of HIV positive patients. Causes for needle biopsy were as follows:

a. Pathologically unknown lymph node enlargement (antiinflammatory treatment is invalid)

b. Clinically suspected lymphatic tuberculosis requiring further clarification

\section{c. History of malignant tumors.}

This study was approved by the Basic research program and Science foundation of the Traditional Chinese Medicine Hospital affiliated to Southwest Medical University and is in accordance with the principle of the Helsinki Declaration II. Informed consent was obtained from each participant.

\section{Method}

The probe frequency using ultrasonic diagnostic apparatus is set to $8 \sim 14 \mathrm{MHz}$ and the semiautomatic biopsy needle included 90 cases of $16 \mathrm{G}$ biopsy needle and 100 cases of $18 \mathrm{G}$ biopsy needle. In the early stages of clinical puncture, routine blood work and blood coagulation examination was performed on the patient. The specific location, number, shape, blood flow characteristics and internal echo of the patient's neck mass were observed and recorded carefully. It was observed whether the probe caused any sub-optimal feeling of pain at the location of the lesion. The patient obtained a supine position and the puncture diagnosis was performed. Color Doppler was used: to analyze the tissue of the lesion area and its peripheral blood flow; to select the best puncture point and method; and to use the $2 \%$ concentration lidocaine hydrochloride after laying the conventional disinfection pad. After anesthesia, ultrasound guided the needle to the edges of the enlarged lymph node, pressed the plug to expose the inner needle, pressed the outer needle automatically under the embolization, and extracted out the tissue to take the groove. In each case, 2 3 needles were used to take out the specimen and then put into sterile filter paper. This was followed by placing it into a $10 \%$ formaldehyde liquid bottle to complete the histopathological examination.

Statistical methods: The data in this paper were processed by SPSS 20.0 software. The measurement data and the calculative data were respectively used and $t$, the calculative data passed the \% test, and the measurement data was analyzed by $x 2$. The data difference was statistically different $(\mathrm{p}<0.05)$.

\section{Results}

The study found 185 patients with satisfactory materials, reaching $97.37 \%$ of the satisfaction rate. No serious complications were found in 90 cases of $16 \mathrm{G}$ biopsy needles and 100 cases of 18G biopsy needles respectively. Of all the patients, only one 23-year-old woman was found to be unsteady at the end of the puncture, and gradually recovered from bed rest. Lymph node lesions were noted to be benign in 115 cases, including 70 cases of lymphatic tuberculosis, 27 cases of reactive hyperplasia, 13 cases of necrotizing lymphadenitis, 4 cases of schwannomas, and 1 case of sarcoidosis. Lymph node lesions in the remaining 75 cases were malignant and included 60 cases of tumor metastasis and 15 cases of lymphoma[8]. Ultrasound and needle biopsy of all patients with benign and malignant cervical lymph node lesions are noted (Tables 1\&2). For cervical lymph node lesions, Ultrasoundguided 16G biopsy achieved $100 \%$ sensitivity, 96\% specificity, and 98\% accuracy whereas Ultrasound-guided 18G biopsy achieved $100 \%$ sensitivity, 94\% specificity, and 97\% accuracy respectively. There was no significant difference noticed between the two needle biopsies in the diagnosis of benign and malignant lesions ( $\mathrm{P}>0.05$ ); 84\% sensitivity, 93\% specificity, and 87\% accuracy were significantly different $(\mathrm{P}<0.05)$, compared with the comparative diagnosis (Table 3).

Table 1: Ultrasound diagnosis of 190 cases of cervical mass.

\begin{tabular}{|c|c|c|c|}
\hline $\begin{array}{c}\text { Ultrasound } \\
\text { Diagnosis }\end{array}$ & Malignant & Benign & Total \\
\hline Malignant & 69 & 6 & 75 \\
\hline Benign & 15 & 100 & 115 \\
\hline Total & 84 & 106 & 190 \\
\hline
\end{tabular}


Table 2: PCNB diagnosis of 190 cases of cervical mass.

\begin{tabular}{|c|c|c|c|c|c|}
\hline \multirow{2}{*}{$\begin{array}{c}\text { Diagnostic } \\
\text { Result }\end{array}$} & \multicolumn{2}{|c|}{ 16G Biopsy needle } & \multicolumn{2}{|c|}{ 18G Biopsy needle } & \multirow{2}{*}{ Total } \\
\cline { 2 - 5 } & Malignant & Benign & Malignant & Benign & \\
\hline Malignant & 32 & 2 & 40 & 2 & 84 \\
Benign & 0 & 54 & 0 & 52 & 106 \\
\hline Total & 32 & 56 & 48 & 54 & 190 \\
\hline
\end{tabular}

Table 3: Comparative diagnostic value of Ultrasound and PCNB diagnosis of 190 cases of cervical mass.

\begin{tabular}{|c|c|c|c|}
\hline $\begin{array}{c}\text { Diagnostic } \\
\text { Index }\end{array}$ & $\begin{array}{c}\text { Ultrasound } \\
\text { Diagnosis }\end{array}$ & $\begin{array}{c}\text { 16G Biopsy } \\
\text { needle }\end{array}$ & $\begin{array}{c}\text { 18G Biopsy } \\
\text { needle }\end{array}$ \\
\hline Sensitivity & 84 & 100 & 100 \\
Specificity & 93 & 96 & 94 \\
Accuracy & 87 & 98 & 97 \\
$\begin{array}{c}\text { Diagnostic } \\
\text { correct index }\end{array}$ & 0.76 & 0.95 & 0.93 \\
\hline
\end{tabular}

(*PCNB: Percutaneous core needle biopsy)

\section{Discussion}

Ultrasound-guided biopsy being easily available and easy to operate has a wide range of applications[5]. It is also considered highly safe. However, due to the significant differences in specific locations of the lesions, the diagnostic value is not uniform[9]. Clinically relevant researchers conducted a study on the diagnostic value of 350 cases of cervical lymph node lesions and found that the satisfaction rate of the material reached $89 \%$. The author believes the satisfaction rate of the material to be closely related to the puncture technique of the clinical operator, as well as the selection of the puncture site and the type of lesion of the patient. The results of this study were satisfactory in 185 patients, reaching $97.37 \%$ of the material satisfaction rate, and all patients had no complications. Ultrasound was able toclearly display the patient's skin and medullary lymph node tissue was completely extracted. For patients with unclear imaging, Color Doppler flow was required to extract a complete material[10]. In patients with lymphadenitis, it was necessary to remove the pus using a $16 \mathrm{G}$ biopsy needle before approaching it, and to select the puncture point as far as possible from the tumor mass. If necessary, surgical resection was required. This study found that ultrasound-guided 18G biopsy achieved $100 \%$ sensitivity, 94\% specificity and $97 \%$ accuracy for cervical lymph node lesions. There was no significant difference between the two needle biopsies in the diagnosis of benign and malignant lesions $(\mathrm{P}>0.05)$. Ultrasound diagnosis reached $84 \%$ sensitivity, $93 \%$ specificity and $87 \%$ accuracy, and there was a significant difference $(\mathrm{P}<0.05)$. Therefore, the needle biopsy is inferred to have a high safety and diagnostic accuracy, as well as a high clinical value.

\section{Conclusion}

Ultrasound-guided percutaneous biopsy can ensure a high accuracy of diagnosis and thereby can reduce the occurrence of complications in patients with cervical lymph node lesions.

\section{Acknowledgement}

This study is partially supported by the Basic Research Program and Science Foundation of The Affiliated Traditional Chinese Medicine Hospital of Southwest Medical University, China. The authors declare no funding and no conflict of interest for this article.

\section{Ethics Approval and Consent to Participate}

The study was approved by Basic research program and Science foundation of the Traditional Chinese Medicine Hospital affiliated to Southwest Medical University.

\section{Human and Animal Rights}

No Animals were used for studies that are base of this research. The reported experiments were in accordance with the ethical standards of the committee responsible for human experimentation (institutional and national), and with the Helsinki Declaration of 1975, as revised in 2008.

\section{Consent for Publication}

Written and informed consents were obtained for the study from all the patients involved in the experiment.

\section{References}

1. Samruddhi Swapnil Metha, Amit Anil Mhapuskar, Swati P Marathe, Swapni Suresh Metha, Santosh Jadhav, et al. (2019) Assessment of efficacy of ultrasonography in cervical lymphadenopathy in oral malignancies. J Family Med Prim Care 8(2): 544-549.

2. Shalva R Gvetadze, Ping Xiong, Jiang Li, Mingming Lv, Jun Li, et al. (2017) Contrast-enhanced ultrasound for diagnosis of an enlarged cervical lymph node in a patient with oropharyngeal cancer: a case report. Oral Surg Oral Med Oral Pathol Oral Radiol 124(5): 495-499.

3. F Mouawad, B Rysman, G Russ, F Benoudiba, G Garcia, et al. (2019) Cystic form of cervical lymphadenopathy. Guidelines of the French Society of Otorhinolaryngology - Head and Neck Surgery (SFORL). Part 1: Diagnostic procedures for lymphadenopathy in case of cervical mass with cystic aspect. Eur Ann Otorhinolaryngol Head Neck Dis 136(6): 489-496.

4. Yu Rong Hong, Zhi YanLuo, Guo Qiang Mo, Ping Wang, Qin Ye, et al (2017) Role of Contrast-Enhanced Ultrasound in the Pre-operative Diagnosis of Cervical Lymph Node Metastasis in Patients with Papillary Thyroid Carcinoma. Ultrasound Med Biol 43(11): 2567-2575.

5. Yan ZHANG, Yukun LUO, Mingbo ZHANG, Ming YANG, Ying ZHANG, et al. (2017) Value of Contrast-enhanced Ultrasound and Conventional Ultrasound in the Diagnosis of Papillary Thyroid Carcinoma with Cervical Lymph Node Metastases 39(2): 177-182.

6. Vincent Balaya, Benedetta Guani, Hélène Bonsang Kitzis, Myriam Deloménie, Charlotte Ngô, et al (2020) Sentinel lymph node biopsy in early-stage cervical cancer: current state of art. Bull Cancer 107(6): 696706.

7. Shogo Shinohara, Shinji Takebayashi, Masahiro Kikuchi, Tetsuhiko Michida, Kazuki Hayashi, et al. (2018) Prognostic impact of incisional or excisional biopsy of cervical lymph node metastases of solid tumors. Jpn J Clin Oncol 48(6): 529-534.

8. Xing Li Yang, Yan Wang, Yong Bao, Shao Bo Liang, Sha Sha He, et al. (2018) Additional Cervical Lymph Node Biopsy is Not a Significant Prognostic Factor for Nasopharyngeal Carcinoma in the Intensity-Modulated Radiation Therapy Era: A Propensity Score-matched Analysis from an Epidemic Area. J Cancer 9(16): 2844-2851. 
9. Jerry Cheng Yen Lai, Ming Shiang Yang, Kuo WeiLu, Lan Yu, Wan Zu Liou, et al. (2018) The role of sentinel lymph node biopsy in early-stage cervical cancer: A systematic review. Taiwan J Obstet Gynecol 57(5): 627-635.

ISSN: 2574-1241

DOI: 10.26717/BJSTR.2020.30.004924

Homagni Sikha Roy. Biomed J Sci \& Tech Res

(c) (P) This work is licensed under Creative

Submission Link: https://biomedres.us/submit-manuscript.php
10. Ariel Gustavo Glickman, Sergio Valdes, Blanca Gil Ibañez, Pilar Paredes, Karen Sttephannía Cortés, et al. (2018) Present status of sentinel lymph node biopsy in cervical cancer. Rep Pract Oncol Radiother 23(6): 495502 .

$\begin{array}{ll}\text { BIOMEDICAL } & \text { Assets of Publishing with us } \\ \text { RESEARCHES } & \text { - Global archiving of articles } \\ \text { - Immediate, unrestricted online access } & \text { - Rigorous Peer Review Process } \\ & \text { - Authors Retain Copyrights } \\ & \end{array}$

